\title{
Optimization Based Spectral Partitioning for Node Criticality Assessment
}

\author{
Waqar Asif* ${ }^{*}$, Marios Lestas ${ }^{\S}$, Hassaan Khaliq Qureshi* and Muttukrishnan Rajarajan ${ }^{\ddagger}$ \\ ${ }^{\ddagger}$ School of Engineering and Mathematical Sciences, City University, London, UK. \\ Email: \{waqar.asif.1, r.muttukrishnan\}@ city.ac.uk \\ * National University of Sciences \& Technology (NUST), Islamabad, Pakistan. \\ Email: \{09mscsewasif, hassaan.khaliq\}@ seecs.edu.pk \\ ${ }^{\S}$ Department of Electrical Engineering, Frederick University, Nicosia, Cyprus. \\ Email: eng.lm@frederick.ac.cy
}

\begin{abstract}
Timely identification of critical nodes is crucial for assessing network vulnerability and increasing network survivability against node failure. In this work, we propose a new distributed algorithm for identifying critical nodes in a network. The proposed approach is based on suboptimal solutions of two optimization problems, namely the algebraic connectivity minimization problem and a min-max network utility problem. The former attempts to address the topological aspect of node criticality whereas the latter attempts to address its connectionoriented nature. The suboptimal solution of the algebraic connectivity minimization problem is obtained through spectral partitioning considerations. This approach leads to a distributed solution which is less computationally expensive than other approaches which exist in the literature and is near optimal, in the sense that it is shown through simulations to approximate a lower bound which is obtained analytically. Despite the generality of the proposed approach, in this work we evaluate its performance on a wireless ad hoc network. We demonstrate through extensive simulations that the proposed solution is able to choose more critical nodes relative to other approaches, as it is observed that when these nodes are removed they lead to the highest degradation in network performance in terms of the achieved network throughput, the average network delay, the average network jitter and the number of dropped packets.
\end{abstract}

\section{INTRODUCTION}

The identification of critical nodes is vital for accessing network vulnerability and security [1]. The failure of a few critical nodes can have an adversarial effect on network performance varying from slight degradation in the Quality of Service up to the complete breakdown of the network [2]. The significance of critical nodes has been highlighted in a number of examples. In Wireless Sensor Networks (WSNs), the energy depletion rate is high at a few nodes rendering them critical for assessing and improving network lifetime [3]. Likewise, the use of clustering in WSNs introduces the risk of eavesdropping by a malicious node on a large amount of information through the clusterheads, making these clusterheads critical for ensuring network privacy [4]. Similarly, in computer networks, the penetration of a virus can be prevented by simply taking a few critical nodes offline, thus ensuring normal network functionality for the rest of the network [5]. Moreover, the effect of a few critical nodes on the overall network connectivity was highlighted in [6], where removal of only $4 \%$ of the nodes in a Peer to Peer Gnutella Network resulted in major fragmentation of the whole network [7]. Finally in a wired telecommunications network, the identification of critical nodes can aid in jamming the network by suppressing the communication between a few critical nodes in the network [8]. The significance of critical nodes goes beyond computer networks to encompass other types of networks such as the transportation network, the power network and the water pipe network. In transportation networks, the identification of critical junctions can aid in forming suitable re-routes in case of an unexpected disruption [8][9]. Similarly, the identification of critical nodes in the power grid network is vital for avoiding network partitioning and large area blackouts [10].

A number of algorithms have been proposed in literature to identify critical nodes in a network. We differentiate between connection based schemes and topology based schemes. Topology based schemes take into account only the underlying topology of the considered network whereas, connection based schemes take into account the users of the network and their source destination paths. Examples of connection based schemes include: the average shortest path length metric [11][12] which calculates the average shortest path length over all possible node destination paths, the closeness centrality metric[13][14], which utilizes the distance of a node to every other node in the network, the rank matrix approach [15], which is based on the effect a node has on the rank of the adjacency matrix of a network upon its removal and the betweenness centrality [16][11][14] and ego centrality [17] metrics, which assess the criticality of a node based on its participation in forming the shortest path routes, with the former using global information and the later taking into account the two hop neighbours. On the other hand, examples of topology based schemes include: the eigenvector centrality metric [18], which uses the largest eigenvector of the adjacency matrix, the Hybrid Interactive Linear Programming Rounding (HILPR) algorithm [10], which analyses the effect that a node has on the pair-wise connectivity of a network upon its removal, the degree centrality metric [13][14] which uses the degree of each node and algebraic connectivity based approaches [19][20][21][22][23] which attempt to minimize 
the algebraic connectivity upon node removal.

Some of these algorithms are based on intuition, whereas others are based on mathematical abstractions of networks of arbitrary topology and are thus characterized by properties which can be verified analytically prior to implementation. In this paper we adopt the latter approach and we cast the node criticality problem in an optimization based framework. We formulate two optimization problems: an algebraic connectivity minimization problem, which addresses the topological aspects of node criticality and a min-max aggregate utility problem which addresses the connection oriented nature of the node criticality. We consider suboptimal solutions for both problems which are combined to yield the proposed criticality identification scheme.

In order to characterize the topological notion of node criticality, we consider a node to be critical when it contributes mostly to keeping the network connected or alternatively when its removal leads to a minimization of the network connectivity. A popular metric which characterizes the connectivity of a network is the algebraic connectivity. The metric was introduced by Fiedler in [19] and is defined as the second smallest eigenvalue of the Laplacian matrix of the network. It has been established in a number of studies [19][24][25] that algebraic connectivity serves as a good measure of connectivity robustness in the sense that the smallest its value is, the closer the network is in becoming disconnected. So, the first optimization problem that we consider in this work is the problem of finding the nodes which, when removed, minimize the algebraic connectivity of the network. A basic but tedious approach to solve the aforementioned problem is to use an exhaustive search over all sub-graphs which result from the removal of each node of the network. This approach assumes knowledge of the entire network topology and can thus become computationally expensive when dealing with large network structures. In addition, when multiple critical nodes need to be found the approach becomes computationally expensive with the number of subgraphs that need to be considered increasing combinatorially with the network size. For this reason, a number of suboptimal solutions have been proposed in literature [22][23][20][21]. These suboptimal solutions utilize the elements of the Fiedler vector which is the eigenvector associated with the second smallest eigenvalue of the Laplacian of the network. Each element of the eigenvector naturally corresponds to a node in the network. The most popular suboptimal node criticality metric is the aggregate squared difference of Fiedler vector elements between neighbouring nodes [22][23] which has been shown to approximate the optimal solution using both analysis and simulations. Recent advances, which allow the distributed calculation of the Fiedler vector values [26] have enabled the distributed implementation of the proposed criticality metric. However, the main drawback of the distributed implementation is that a global maximisation consensus algorithm must be employed which can be slow and significantly increases the convergence time.

In this work we adopt an alternative approach to obtaining a suboptimal solution of the original algebraic connectivity minimization problem by employing spectral partitioning concepts. It is well known that the elements of the Fiedler vector assume positive and negative values in the range $[-1,1]$ and that a splitting value $s$ can be used to partition the network in two clusters (the first cluster containing all the nodes with corresponding Fiedler vector values less than $s$ ). Different values of $s$ yield different types of cuts such as bisection, ratio cut, sign cut and gap cut [19]. The Fiedler clusters are known to be well connected [26] and in addition it has been shown that for various types of networks they possess the desired property that they have nearly equal number of vertices with minimum number of edges in-between them [25]. In this work, based on the latter property, we consider as critical, the nodes which lie on the boundary of the Fiedler clusters. We adopt the sign cut approach and we thus consider as critical, the nodes which have at least one neighbour with a corresponding Fiedler vector value of different sign. This approach is attractive to be implemented in a distributed manner and allows each node to decide by itself whether it is a critical node. In addition, we demonstrate that this approach is directly related to the approach in [22][23] as the nodes which lie on the boundary of the Fiedler clusters report high values of the aggregate squared Fiedler vector value differences, which is the criticality metric proposed therein. However, when a single critical node is required and a maximization algorithm needs to be employed, the proposed algorithm offers the advantage that it significantly reduces the distributed computational complexity as the maximization algorithm needs to be applied only over a reduced set of nodes, namely the ones which have the same Fiedler vector element sign. We demonstrate through simulations the significant reduction in convergence time achieved, and in addition we show that the solution is near optimal, in the sense that it approximates to a very good extent, a lower bound on the achieved algebraic connectivity which we derive analytically.

As pointed out above, the proposed change of sign method can lead to multiple nodes being detected as critical and so, when a single node is required, a metric must be utilized to decide on the most critical node among the ones which lie on the boundary of the Fiedler clusters. In our recent work in [27], we have adopted the metric in [22][23], however, in this work we consider an alternative metric which takes into account the users of the underlying network and their source destination paths. The algebraic connectivity depends only on the topology of the underlying network and the criticality metric must thus be complemented to account for the intuitive notion that the users of the network must also be taken into consideration when assessing the criticality of a node. This complementary information is offered by the second optimization problem that we consider in this work. It has been well established in the literature that the rate allocation algorithms of the network users attempt to maximize the aggregate utility of the network over the capacity constraints [28]. So, we consider as critical, the nodes which, when removed degrade the network performance to the greatest extent i.e. they minimize the maximum of the aggregate utility function. This optimization problem requires full network information in order to be solved and in addition the complexity of the exhaustive search solution increases combinatorially with the network size when multiple nodes need to be selected. We thus derive a suboptimal solution which identifies as critical, the nodes which maximize the 
square root of the number of active connections at each node multiplied by the aggregate input data rate. We thus combine the suboptimal solutions of the two optimization problems to derive the proposed criticality metric which considers as critical the nodes which maximize the latter criticality metric over the nodes which lie on the boundary of the Fiedler clusters.

We evaluate the performance of the proposed criticality metric using extensive simulations conducted on Matlab and the Ns-3 simulator. Since the criticality metric is obtained by combining suboptimal solutions of two optimization problems we first establish that these suboptimal solutions are not conservative. When a single critical node is removed, the proposed suboptimal solutions are very close to the optimal ones which are obtained using the exhaustive search approach. When multiple nodes are removed the suboptimal solutions are close to a lower bound which is obtained analytically. We then compare the proposed metric against other metrics which have been proposed in literature: the Betweenness Centrality [16], the Closeness Centrality, the Degree Centrality [13], the Hybrid Interactive Linear Programming Rounding (HILPR) proposed in [10], the Controllability of complex networks (Cont) in [15], the suboptimal solution of Eq (6) [20][21] and the suboptimal solution of Eq (7) [22][23]. The evaluation is based on the degradation in performance reported when nodes selected using the criticality metrics under consideration are removed from the network. The considered network is a wireless ad-hoc network where the $\mathrm{x}$ and $\mathrm{y}$ coordinates of the nodes are randomly chosen according to uniform distributions. We establish that the proposed criticality metric outperforms the other approaches in terms of the achieved network throughput, the average network delay, the average network jitter and the number of dropped packets.

The rest of the paper is organised as follows. In Section II, we introduce the relevant mathematical framework and formulate the considered optimization problems, in Section III, we present the suboptimal solutions of the formulated optimization problems which are combined to yield the proposed criticality metric, in Section IV, we derive the lower bound on the algebraic connectivity upon node removal, in Section V, we evaluate the performance of the proposed approach using simulations and finally in Section VI we conclude our work.

\section{PROBLEM FORMULATION}

The proposed method for identifying critical nodes is based on the solution of two optimization problems: the algebraic connectivity minimization problem and a min-max aggregate utility problem. In this section we introduce the relevant mathematical framework which is used to formulate these problems mathematically and also present some of the relevant approaches present in the literature.

\section{A. Algebraic Connectivity Minimization}

We consider an undirected graph $G=(V, E)$ where $|V|=n$ and $|E|=m$ are the number of nodes and edges respectively. The existence of an edge $l \in E$ between nodes $i$ and $j$ defines the $l^{t h}$ column $a_{l}$ of the incidence matrix $A \in \Re^{n \times m}$ of the graph such that $a_{l_{i}}=1, a_{l_{j}}=-1$ and 0 otherwise. The Laplacian matrix is then defined as:

$$
L=A A^{T}=\sum_{l=1}^{m} a_{l} a_{l}^{T}
$$

$L$ is positive semi-definite and thus has an orthonormal basis of real eigenvectors with corresponding real eigenvalues. It also holds that $L \mathbf{1}=0$ where $\mathbf{1}$ is the vector of all ones. The latter property guarantees that at least one eigenvalue of the Laplacian matrix is equal to zero. We rearrange the eigenvalues of the Laplacian matrix in ascending order such that $0=\lambda_{1} \leq \lambda_{2} \leq \ldots \leq \lambda_{n}$. The second smallest eigenvalue $\mu=\lambda_{2}$ is known as the algebraic connectivity of the graph with the corresponding normalized eigenvector being referred to as the Fiedler vector [19].The algebraic connectivity is related to the connectivity of the graph as a result of the following theorem [29].

\section{Theorem 1. $G=(V, E)$ is disconnected if and only if $\mu=0$}

Further, the algebraic connectivity has been observed to serve as a connectivity robustness measure in the sense that the lower its value is, the closer the network is in becoming disconnected. The latter property has motivated the use of the algebraic connectivity in assessing node criticality. A node is considered to be critical when it contributes mostly to keeping the network connected. One may thus define as critical, the nodes which when removed minimize the algebraic connectivity of the network. This optimization problem, referred to as optimization problem $P$, is shown formally below:

$$
P: C N=\arg \min _{\alpha \in V} \mu(G(V-\alpha))
$$

One way of solving $P$ when a single node is removed is through exhaustive search. However this approach is computationally expensive. In addition, when multiple nodes are removed, the complexity of the exhaustive search solution increases combinatorially with increasing network size. So, people have sought suboptimal solutions which are simple to implement in a distributed manner. The most popular solutions are inspired from the following characterization of the algebraic connectivity [30] using the Rayleigh quotient of $y$ with respect to $\mathrm{L}$ :

$$
\mu(L)=\min \left\{\frac{y^{T} L y}{y^{T} y} \mid y \neq 0, \mathbf{1}^{\mathbf{T}} y=0\right\}
$$

If we substitute $y$ with the normalized vector $v=y /\|y\|$ in Eq (3) then, it can be written as:

$$
\mu(L)=\min \left\{v^{T} L v \mid\|v\|=1, \mathbf{1}^{\mathbf{T}} v=0\right\}
$$

which can also be expressed in the form:

$$
\mu(L)=\min \left\{\sum_{i=1}^{n} \sum_{j \in N_{i}}\left(v_{i}-v_{j}\right)^{2} \mid\|v\|=1, \mathbf{1}^{\mathbf{T}} v=0\right\}
$$

where $N_{i}$ is the set of neighbours of node $i$. The minimum is achieved when $v$ is the Fiedler vector of the Laplacian $L$. 
Each Fiedler vector entry naturally corresponds to a node in the graph. It can thus be deduced from equation (5) that the node which contributes the most to the algebraic connectivity is the one with the maximum sum of squared Fiedler vector value differences with neighbouring nodes i.e $\sum_{j \in N_{i}}\left(v_{i}-v_{j}\right)^{2}$. Based on this, the authors in [20], [21] have considered as critical, the nodes which solve the following optimization problem:

$$
C N=\arg \max _{i \in V} \sum_{j \in N_{i}}\left(v_{i}-v_{j}\right)^{2}
$$

The variant shown below has also been proposed in [22] and $[23]$

$$
C N=\arg \max _{i \in V} \frac{\sum_{j \in N_{i}} v_{j}\left(v_{i}-v_{j}\right)}{1-v_{i}^{2}}
$$

The solutions of (6) and (7) constitute suboptimal solutions of the optimization problem $P$ as indicated in [22]. As a result of recent advances in the distributed calculation of Fiedler vector values [26], these suboptimal solutions are amenable for implementation in a distributed manner. The main drawback of the distributed implementations, as indicated by the authors in [22][21], is that, a maximization consensus algorithm must be employed over the entire set of nodes present in the relevant graph which increases significantly the computational overhead. In this work, we offer an alternative suboptimal solution which alleviates the aforementioned problem thus reporting smaller convergence times.

\section{B. Min-Max Aggregate Utility}

The algebraic connectivity, which has so far been used to assess node criticality, only takes into account the topology of the underlying network. However, intuition suggests that apart from the network topology, the network users also have a key role to play when assessing the criticality of a particular node. Nodes which are utilized by many source destination paths, or nodes which accommodate large amounts of data traffic, can be considered more critical than others. In this section, we utilize the Network Utility Maximization (NUM) framework proposed by Kelly in [31] to cast these intuitive notions in a formal optimization based framework.

We consider a network which consists of a set of traffic sources $S$ and a set of links $L$. Each network user $s \in S$ injects data into the network with a rate denoted by $x_{s}$. The data is transferred from its source $s \in S$ to its destination via a route which comprises of a set of links collected in the set $L(s)$ representing the route. Each link $l \in L$ is characterized by a finite capacity $c_{l}$. To each user $x_{s}$ we assign a utility function $U_{s}\left(x_{s}\right)$ which represents the satisfaction a user gets from a particular sending rate allocation. The utility functions are assumed to be strictly increasing, continuously differentiable and strictly concave. The objective of the network user collaboration is then to maximize the aggregate utility function subject to the capacity constraints. So, a node is considered to be critical if its removal has the greatest negative impact on the aforementioned objective i.e. it minimizes the maximum feasible aggregate utility function subject to capacity and feasibility constraints. This is expressed formally below:

$$
\begin{gathered}
Q: C N=\arg \min _{k \in V_{c}} \max _{s \in(S \backslash k)} \sum_{s} U_{s}\left(x_{s}\right) \\
\text { subject to } \sum_{s: l \in(L(s) \backslash L(k))} x_{s} \leq c_{l} \forall l \\
\text { over } x_{s} \geq 0
\end{gathered}
$$

The optimization problem of $\mathrm{Eq}$ (8) is a mixed integer discrete continuous problem, discrete in the minimization over the set of nodes and continuous in the maximization over the sending rates. One may employ the exhaustive search approach to obtain the optimal solution when a single node is removed. However, this approach is computationally expensive and requires full network information. In addition, when multiple nodes are removed the complexity of the exhaustive search approach increases combinatorially with network size. A number of algorithms have been proposed in literature to obtain more efficient optimal and suboptimal solutions [32][33]. In this work we offer, a suboptimal solution which leads to a distributed, simple to evaluate node criticality metric.

\section{PROPOSED Algorithm}

In this section, we describe the proposed criticality metric which is based on suboptimal solutions of the optimization problems $P$ and $Q$ described in the previous sections. The rationale behind the offered suboptimal solutions is explained.

\section{A. Algebraic Connectivity Minimization}

The proposed suboptimal solution of problem $P$ is based on spectral partitioning considerations. Spectral partitioning, refers to the methodology with which a graph can be partitioned into connected clusters using spectral properties of the graph, namely the elements of the Fiedler vector. As a result of the property $\mathbf{1}^{\mathbf{T}} v=0$ in (5) the elements of the Fiedler vector attain both positive and negative values in the range $[-1,1]$. The following theorem establishes how the Fiedler vector elements can be used to partition the graph into clusters which are well connected [29].

Theorem 2. Let $G$ be a finite connected graph with $N$ vertices and $v_{i}$ be the Fiedler vector value corresponding to node $i$. Then for any $s \geq 0$ :

$$
M(s)=\left\{i \in N \mid v_{i}+s \geq 0\right.
$$

the sugbraph $G(s)$ induced by $G$ on $M(s)$ is connected.

A similar theorem exists for $s \leq 0$. Different values of $s$ yield different types of cuts [29]. In this work we adopt the sign cut approach in which case $s$ is equal to 0 . The above theorem only establishes the connectivity of the obtained clusters. However, a number of other results indicate that spectral partitioning can produce cuts with a good ratio of cut edges to separated vertices [34]. This implies that spectral partitioning methods yield strongly connected clusters of approximately equal size, loosely connected between them. This property 
motivates the proposed solution. As we are looking for nodes which when removed minimize the algebraic connectivity we expect that if an edge lying in the spectral partitioning cut-set is removed from the network, it will render the clusters even less loosely connected thus significantly decreasing the algebraic connectivity of the network. We thus consider as critical the nodes whose removal will result in the removal of an edge from the spectral partitioning cut-set. As mentioned above, in this work we adopt the sign cut approach which partitions the network into two well-connected clusters. All the nodes of the first cluster have positive corresponding Fiedler vector elements whereas, all the nodes of the second cluster have negative Fiedler vector elements. The cut-set thus comprises of all the edges which connect nodes with corresponding Fiedler value elements of different sign. We thus consider as critical the nodes which have at least one neighbouring node with a Fiedler vector element of different sign. In mathematical terms a node $i \in V$ is critical if it satisfies:

$$
\sum_{j \in N_{i}} \operatorname{sign}\left(\left|v_{i}-v_{j}\right|\right)>0
$$

where sign is the sign function and $v_{i} \in V$ are the elements of the Fiedler vector.

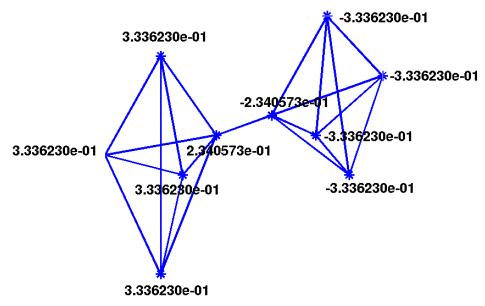

Figure 1. Example network where the Fiedler values are indicated at the corresponding nodes.

We demonstrate these concepts through the sample network of Fig 1. The network consists of two well connected subgraphs. These are loosely connected between them by means of a single link. The Fiedler vector values are calculated and indicated on the diagram. We observe that the Fiedler vector values corresponding to the nodes in the left-hand subgraph have positive values, whereas, the elements corresponding to nodes in the right-hand subgraph have negative values. Intuition suggests that the nodes which are critical are the ones which connect the two subgraphs via the single edge. We observe that these two nodes have Fiedler vector values of different signs and are thus correctly detected by the proposed criterion of equation (12).

The question that arises is whether the proposed criterion is indeed a suboptimal solution of the algebraic minimization problem $P$ in (2). In subsequent sections we demonstrate the suboptimality using simulations. In this section we demonstrate the suboptimality by highlighting its relation to the criticality criterion in (6) which has been demonstrated [20] to constitute a suboptimal solution. In particular, we show that the nodes which are detected as being critical according to the proposed criterion of equation (12) also report high aggregate squared

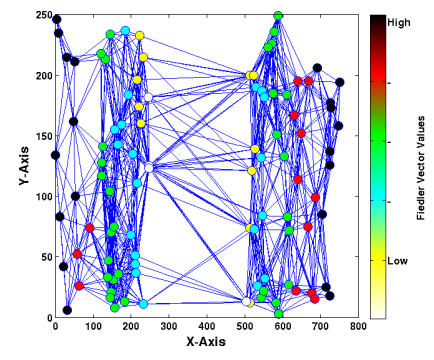

(a)

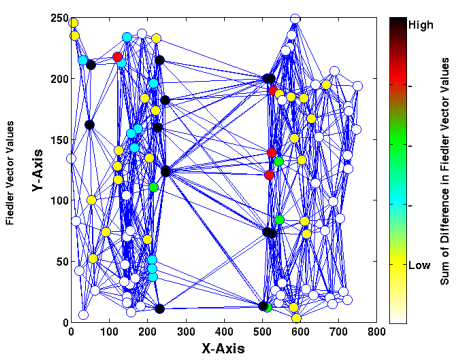

(b)
Figure 2. Example network where at each node we highlight a) Fiedler vector values, b) Difference in Fiedler vector value across the network.

Fiedler difference values $\sum_{j \in N_{i}}\left(v_{i}-v_{j}\right)^{2}$ which implies that they are also critical according to criterion (6). The analytical verification of this observation is an open problem. This observation is important as it suggests that the maximization of (6) does not have to be done over the entire set of nodes but only over the ones which have Fiedler element values of the same sign. This can significantly reduce the implementation complexity of (6).

To demonstrate the relationship we use the network of Fig. 2 which comprises 80 nodes. The network consists of two well connected subgraphs loosely connected by a small set of edges. Each node is coloured according to the magnitude of the absolute value of the quantity under investigation. In Fig. 2(a) we show at each node $i$ the magnitude of the calculated Fiedler element value $v_{i}$ whereas, in Fig. 2(b) we show the magnitude of the aggregate squared difference value $\sum_{j \in N_{i}}\left(v_{i}-v_{j}\right)^{2}$. We observe that there is a tendency for the Fiedler elements to attain their lowest value at nodes which lie in the sign cutset. As we move away from the sign cut-set the Fiedler values tend to increase. In addition, we observe that nodes which lie in the sign cut-set tend to attain large aggregate squared difference values. This demonstrates the relationship between (12) and (6).

The proposed change of sign approach is amenable for implementation in a distributed manner. Recent techniques [26], allow the distributed calculation of Fiedler values at each node. Then, the only thing that a node needs to do in order to classify itself as critical is to check whether at least one of its neighbours has a Fiedler value with a different sign than itself. However, this approach leads to multiple nodes being detected as critical. What if a single node needs to be selected? Among the nodes which lie in the sign cut-set how do we choose the one which is the most critical? In our recent work in [27] we have chosen the node which maximizes the sum of squared differences $\sum_{j \in N_{i}}\left(v_{i}-v_{j}\right)^{2}$. In this work, we choose an alternative criterion which is based on a distributed suboptimal solution of the problem $Q$ in (8).

\section{B. Min-Max Aggregate Utility}

We relax the strict concavity of the utility functions to assume linear utility functions $U_{s}\left(x_{s}\right)=x_{s}$. We consider two approaches to obtain suboptimal solutions which are combined to obtain the proposed criticality metric. The first approach 
is via the directional derivative along the directions of rate deductions due to link removal. Let $F\left(x^{*}\right)=\sum_{s} U_{s}\left(x_{s}^{*}\right)$ denote the aggregate utility function evaluated at the optimal sending rates at which the maximum is achieved. When a link $l \in L$ is removed from the network then all the sources $s$ which utilize link $l$ denoted by $S(l)$ will be deprived from the ability to send data. We thus investigate the effect of removing link $l$ on $F$ by considering the directional derivative of $F$ along the unit vector $\overrightarrow{y_{l}}=\sum_{s \in S(l)} \frac{1}{\sqrt{n_{l}}} \overrightarrow{i_{s}}$, where $n_{l}$ is the cardinality of $S(l)$ and $\overrightarrow{i_{s}}$ is the unit vector along the direction $x_{s}$. The directional derivative evaluated at the equilibrium point $x^{*}$ is given by

$$
\begin{array}{r}
D_{\overrightarrow{y_{l}}} F=\overrightarrow{y_{l}} \cdot \nabla f_{x=x^{*}}= \\
\frac{1}{\sqrt{n_{l}}} \frac{\partial F}{\partial x_{1}}+\frac{1}{\sqrt{n_{l}}} \frac{\partial F}{\partial x_{2}}+\ldots+\frac{1}{\sqrt{n_{l}}} \frac{\partial F}{\partial x_{n}}
\end{array}
$$

Since the utility functions are assumed linear:

$$
D_{\overrightarrow{y_{l}}} F=\sqrt{n_{l}}
$$

Since the objective is to minimize $F\left(x^{*}\right)$ links $l$ are sought which maximize the directional derivative. The other approach is by direct calculation of the reduction in $F\left(x^{*}\right)$ when a link $l$ is removed. Due to the linear utility function assumption, $F\left(x^{*}\right)=\sum_{s \in S} x_{s}^{*}$. When a link $l$ is removed, all the sources $s$ which utilize link $l$ will be deprived from the ability to send data. This will result in a reduction in $F\left(x^{*}\right)$ by an amount $\Delta y_{l}^{*}=\sum_{s \in S(l)} x_{s}^{*}$. Since the objective is to minimize $F\left(x^{*}\right)$, links $l$ are sought which report the highest input data rate $\Delta y_{l}^{*}$. We combine the aforementioned approaches to classify as critical the links which satisfy:

$$
C N=\arg \max _{l \in L} \sqrt{n_{l}} \Delta y_{l}^{*}
$$

Despite the fact that the discussion has so far been made with reference to link removal, the derived criterion of equation (15), also applies to node removal. $n$ is the total number of connections traversing the node, whereas $\Delta y^{*}$ is the input data rate at the node. The dependence of the criticality metric on $n$ is in line with the well known betweenness centrality criterion. The dependence on the input data rate is in line with the intuitive notion that the more data traverses a node the more critical it is. The input data rate at a particular node is a quantity that can be calculated locally. The number of active connections, however, is readily available locally only in systems which maintain per connection states at each node. When such per connection states are not available, estimates of the active connections can be used instead. Such estimates can be generated online using parameter identification techniques proposed in literature [35].

The obtained suboptimal solutions of the two considered optimization problems are then combined to yield the methodology with which the most critical node in the network is identified. The methodology is as follows. The change of sign approach of equation (12) is first used to identify all the nodes which lie in the sign cut-set. Among the nodes which lie in the sign cut-set, the most critical is the one which maximizes the cost function of (15). The proposed approach is amenable for implementation in a distributed manner. Recently proposed techniques [26] allow the distributed calculation of the Fiedler elements at each node. After the Fiedler elements are calculated at each node, the nodes employ beacon message exchange to share their Fiedler elements with their neighbours. If a node detects that the sign of the Fiedler value of one of its neighbours is different than its own sign, then it identifies itself as lying in the sign cut-set of the network graph. All the nodes that lie in the sign cut-set calculate the $\sqrt{n} \Delta y$ cost of equation (15) and initiate a blind flooding algorithm to share their cost with all the other nodes lying in the sign cut-set. When a node in the sign-cut set receives a cost initiated from another node in the sign cut-set it compares the two, and if the maximum is its own cost it identifies itself as a critical node and rebroadcasts the maximum of the two. This approach guarantees that when the algorithm terminates, only one critical node is left within the network which is the one which has the highest cost among all the nodes which lie in the sign cut-set. Note that the blind flooding algorithm is implemented only over the nodes which share the same Fiedler element sign. This achieves significant savings in computation effort relative to other approaches. Below, we show a pseudocode of the proposed method.

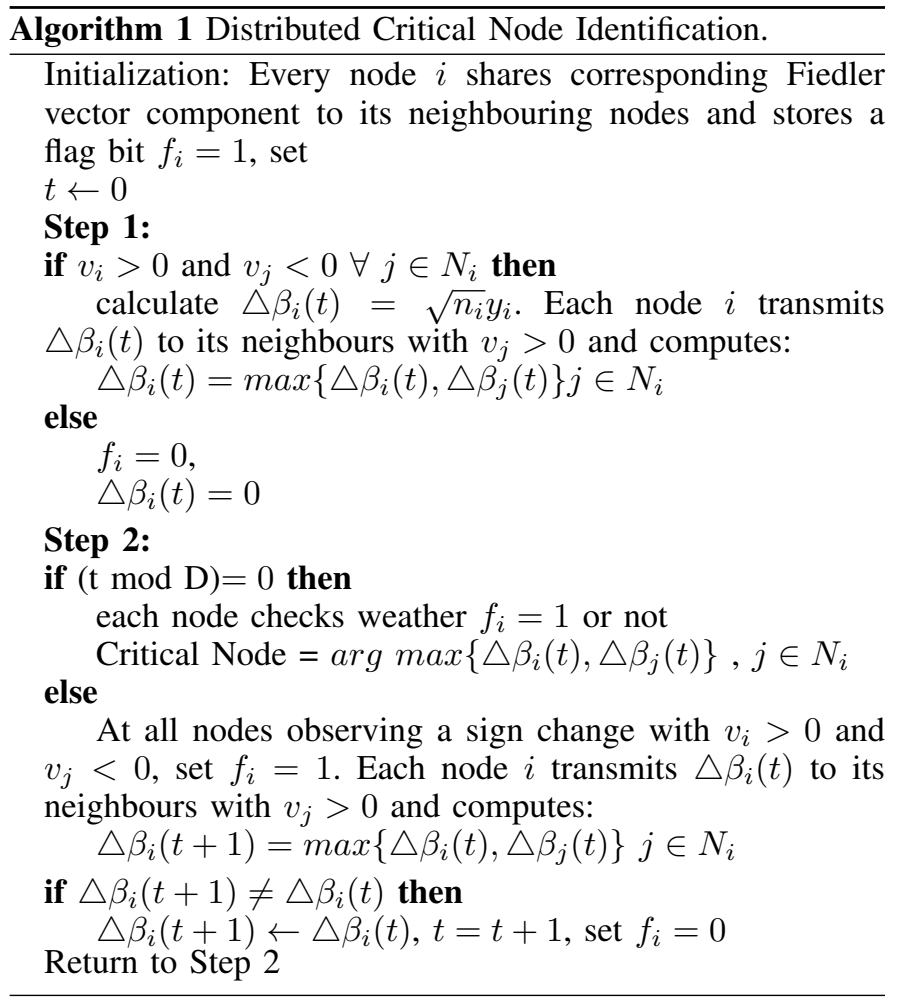

In the future we aim at finding more efficient distributed algorithms which take into account the fact that the Fiedler values are minimum at the nodes which lie in the sign cut-set. 


\section{ANALYSIS}

In this section, we derive analytically a lower bound on the algebraic connectivity when multiple nodes are removed from the network under consideration. This bound allows one to evaluate how conservative our suboptimal solutions are when multiple nodes are removed from the network.

Theorem 3. Let $G=(V, E)$ be a graph of $n$ nodes with eigenvalues $0 \leq \wedge_{2} \leq \wedge_{3} \leq \ldots \leq \wedge_{n}$. Then, upon removal of $w$ nodes from the graph, the algebraic connectivity of the resultant graph is lower bounded by:

$$
\lambda \geq \wedge_{2}-\frac{u_{2}^{2}}{1+\left(b_{n}-u_{2}^{2}\right) /\left(\wedge_{2}-\wedge_{n}\right)}
$$

where

$$
\begin{gathered}
u_{2}=\sum_{w \in n} \sum_{j \in N_{i}, i \in w}\left(v_{i}-v_{j}\right), \\
b_{n}=n\left(\operatorname{tr}(A)-u_{2}\right)+\sqrt{n(1-n) f(A)}
\end{gathered}
$$

and

$$
f(A)=\operatorname{tr}\left(A-\frac{\operatorname{tr}(A)}{2} I\right)^{2}-\left(2\left(u_{2}-\frac{\operatorname{tr}(A)}{2}\right)^{2}\right)
$$

with

$$
\operatorname{tr}\left(A-\frac{\operatorname{tr}(A)}{2} I\right)^{2}=\operatorname{tr}\left(A^{2}\right)-\frac{(\operatorname{tr}(A))^{2}}{2}
$$

Here, $A$ is the Laplacian matrix defined by the set of nodes $w$ that are being removed from the graph.

Proof: We use the eigenvalue decomposition of $L=$ $Q D Q^{T}$ where $D=\operatorname{Diag}\left(0, \wedge_{2}, \ldots ., \wedge_{n}\right)$ is the diagonal matrix of ascending eigenvalues and $Q$ is an orthogonal matrix with corresponding eigenvectors of $L$ in its columns. The eigenvalues of a Laplacian matrix $L$ can be found using $L v=\lambda v$, therefore, in this expression we substitute $L$ to get [36]:

$$
\left(Q D Q^{T}\right) v_{j}=\wedge_{j} v_{j}
$$

Where $v_{j}$ is the linear combination of the eigenvectors corresponding to the $j^{\text {th }}$ eigenvalue $\wedge_{j}$ of $L$. The removal of $w$ nodes from the network reduces $D$ by a factor $u u^{T}$ where $u=Q^{T} H$ and $H$ is the incidence matrix defined by the set of nodes being removed [37]. Thus we have:

$$
Q\left(D-u u^{T}\right) Q^{T} v_{j}=\wedge_{j} v_{j}
$$

We know from [38] that, the eigenvalues of Eq (22) can be obtained by solving $D-u u^{T}-\lambda I$ for the determinant of the matrix, where $I$ is the identity matrix [38]:

$$
\begin{gathered}
\operatorname{det}\left(D-u u^{T}-\lambda I\right)=0 \\
\operatorname{det}(D-\lambda I) \operatorname{det}\left(I-(D-\lambda I)^{-1} u u^{T}\right)=0
\end{gathered}
$$

Eq (24) can be reduced to [38]:

$$
\prod_{i=1}^{n}\left(\wedge_{i}-\lambda\right)\left(1-\sum_{i=1}^{n} \frac{u_{i}^{2}}{\left(\wedge_{i}-\lambda\right)}\right)=0
$$

This shows that, the eigenvalue of Eq (22) can be computed by finding the roots of the secular equation:

$$
1=\sum_{i=1}^{n} \frac{u_{i}^{2}}{\bigwedge_{i}-\lambda}
$$

We solve Eq (26) for the the eigenvalue $\lambda$ of the network that results after the removal of $w$ node from the network. Here, we know that $u_{1}=0$ and $u_{2}=\sum_{w \in n} \sum_{j \in N_{i}, i \in w}\left(v_{i}-v_{j}\right)$. Therefore we have:

$$
\frac{u_{2}^{2}}{\wedge_{2}-\lambda}=1-\sum_{i=3}^{n} \frac{u_{i}^{2}}{\bigwedge_{i}-\lambda}
$$

This can be re-arranged into:

$$
\lambda=\wedge_{2}-\frac{u_{2}^{2}}{1+\sum_{i=3}^{n} u_{i}^{2} / \lambda-\wedge_{i}}
$$

According to the eigenvalue interlacing theorem, the algebraic connectivity of network that results from the removal of a node is bounded by $0 \leq \lambda_{2} \leq \wedge_{2}$ [39].

Theorem 4. Let $X$ be a graph with $n$ vertices and let $Y$ be obtained by removing a vertex from $X$ then [39]:

$$
\lambda_{i-1}(L(X)) \leq \lambda_{i}(L(Y)) \leq \lambda_{i}(L(X))
$$

We use Theorem 4 along with the observation in Eq (28), that the LHS is a decreasing function whereas the RHS is an increasing function of $\lambda$, therefore we obtain the lower bound of $\lambda$ by using the appropriate substitution of $\lambda=\wedge_{2}>\lambda_{2}$. This gives us:

$$
\lambda \geq \wedge_{2}-\frac{u_{2}^{2}}{1+\sum_{i=3}^{n} u_{i}^{2} /\left(\wedge_{2}-\wedge_{n}\right)}
$$

From [40] we know that $\sum_{i=1}^{n} u_{i}^{2} \leq b_{n}$, thus we approximate $\sum_{i=3}^{n} u_{i}^{2}$ with the difference $b_{n}-u_{2}^{2}$ to obtain the final expression of Eq (16), where:

$$
b_{n}=n\left(t r(A)-u_{2}\right)+\sqrt{n(1-n) f(A)}
$$

and $f(A)$ is:

$$
f(A)=\operatorname{tr}\left(A-\frac{\operatorname{tr}(A)}{2} I\right)^{2}-\left(2\left(u_{2}-\frac{\operatorname{tr}(A)}{2}\right)^{2}\right)
$$

In Eq (31) the square of the matrix can be avoided by using Eq (32) [40].

$$
\operatorname{tr}\left(A-\frac{\operatorname{tr}(A)}{2} I\right)^{2}=\operatorname{tr}\left(A^{2}\right)-\frac{(\operatorname{tr}(A))^{2}}{2}
$$

Here $\operatorname{tr}\left(A^{2}\right)=\|A\|_{f}^{2}$ and $\|A\|_{f}$ is the Frobenius matrix norm of $\mathrm{A}$. 


\section{PERformance Evaluation}

In this section, we evaluate the performance of the proposed criticality metric using simulations conducted on Matlab [41] and on the Network Simulator (Ns-3) [42]. We first assess how conservative the suboptimal solutions are with reference to the posed optimization problems, and we then evaluate the ability of the proposed method to choose the most critical nodes in the network. The criticality of a fixed number of nodes is assessed by evaluating the degradation in performance achieved when these nodes are removed from the network. We conduct a comparative study to investigate the performance of the proposed metric against other approaches that exist in literature such as the Betweenness Centrality [16], the Closeness Centrality, the Degree Centrality [13], the exhaustive search approach, the Hybrid Interactive Linear Programming Rounding (HILPR) metric proposed in [10], the controllability of complex networks (Cont) approach in [15], the suboptimal solution of $\mathrm{Eq}$ (6) which we refer to as the Sum Squared Difference approach (SSD) [20][21], the suboptimal solution of Eq (7) which we refer to as the Normalized Sum Squared Difference approach (NSSD) [22][23] and our previously proposed approach which we refer to as Spectral Partitioning for Node Criticality approach (SPNC) [27]. Our simulation results indicate that the suboptimal solutions are not conservative and that the proposed criticality metric chooses the most critical nodes in the network as it achieves the greatest degradation in performance when these nodes are removed.

\section{A. Algebraic Connectivity Suboptimality}

In this section we evaluate, using simulations conducted on Matlab, the ability of the change of sign approach, incorporated in the proposed metric, to serve as a suboptimal solution of the posed algebraic connectivity minimization problem i.e. to identify nodes which when removed achieve algebraic connectivity values which are close to the minimum. We also compare the change of sign approach with other approaches which have been proposed in literature in terms of the algebraic connectivity achieved. As our objective is to focus on the topological aspects of the proposed criticality metric we do not account for network users. To find a single critical node we first employ the change of sign approach to find the set of nodes which lie in the sign cut-set and among these we find the one which maximizes the $\sqrt{n_{l}}$ parameter. The parameter $n_{l}$ at a particular node $l$ is found by calculating the number of times the node $l$ participates in the shortest path, among all shortest paths between all possible source destination pairs.

We consider an area of $1000 \times 1000 m^{2}$ in which we randomly deploy 100 nodes. The $x$ and $y$ coordinates of the nodes are drawn from a uniform random distribution. The nodes employ wireless communication to form a wireless ad hoc network. In order to evaluate the performance of the considered criticality metrics as a function of the transmission radius of the nodes, we consider transmission radius values in the range $100 \mathrm{~m}$ to $200 \mathrm{~m}$. To avoid random fluctuations due to single simulation run, simulations were conducted for 20 different network topologies and the results were then averaged.

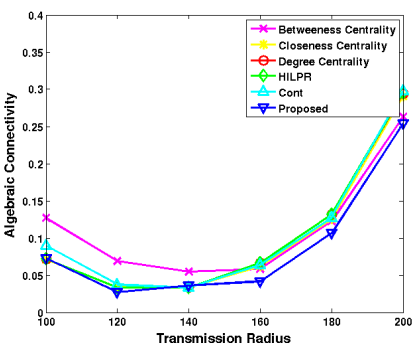

(a)

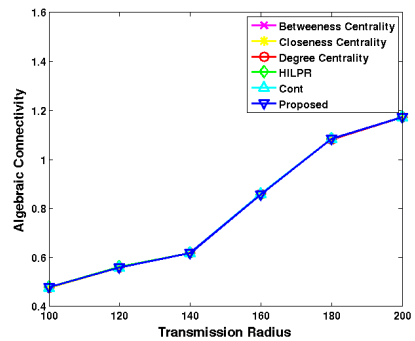

(c)

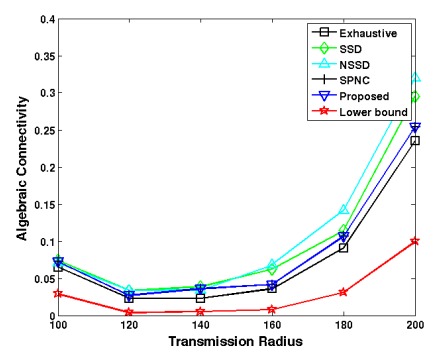

(b)

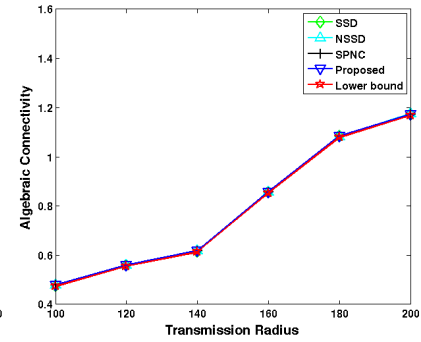

(d)
Figure 3. Algebraic Connectivity versus the trasnsmission radius when: a) \& b) a single node is removed from the network, c) \& d) five nodes are removed from the network.

In Fig 3a \& 3b, we show the algebraic connectivity of the aforementioned network as a function of the transmission radius when only one node, the most critical in the network, is removed. In each case we remove a node using a different criticality metric. We compare the proposed change of sign approach against the exhaustive search approach, the betweenness centrality, the closeness centrality, the degree centrality, the HILPR, the Cont,the SSD and the NSSD. Note that when a single node is removed the optimal algebraic connectivity value can be found using the exhaustive search approach i.e. the algebraic connectivity is calculated when each node is removed from the network and the minimum among all calculated values is recorded.

The first thing to note is that, as expected, the algebraic connectivity increases monotonically as the transmission radius increases. The other thing to note is that at almost all transmission range values, the proposed change of sign approach, manages to yield the smallest algebraic connectivity value which is surprisingly very close to the optimal value calculated using the exhaustive search approach. This demonstrates that the proposed suboptimal solution is not conservative in the sense that it yields algebraic connectivity values which are close to the optimal. We next conduct a similar evaluation study when 10 nodes are removed from the network. When multiple nodes are removed the exhaustive search approach becomes computationally expensive, so in order to evaluate the suboptimality of the proposed approach we compare it with the lower bound calculated in section IV. The results are shown in Fig 3c \& 3d. The results indicate that all criticality metrics report similar algebraic connectivity values which are close to the lower bound. This again demonstrates the fact that the proposed suboptimal solution is not conservative. 


\section{B. Network Utility Maximization Suboptimality}

The main objective of this set of simulation experiments is to evaluate how conservative the proposed criticality metric is in solving the min-max optimization problem (8). Since a suboptimal solution is proposed, it is crucial to evaluate the degree with which the metric identifies nodes which when removed lead to aggregate utility functions which are close to the optimal. The optimal cost function is found by employing an exhaustive search approach i.e. the maximum aggregate utility is calculated, when each node is removed from the network and the minimum is found among all values calculated. We consider logarithmic utility functions which are common in the literature. In our simulation experiments we consider an area of $100 \times 100 m$ where we deploy 50 nodes with the $x$ and $y$ coordinates drawn from a uniform random distribution. Each node is characterized by a transmission radius of $30 \mathrm{~m}$. At each time instant, a particular number of users inject data into the network along specific data routes. We vary the number of users from 5 to 20 and the reported results are averaged over 50 experiment repetitions, in order to decrease the inaccuracies due to the random nature of the setting. As the proposed criticality metric incorporates the number of users traversing the node which is related to the betweenness centrality metric, we compare the proposed approach not only with the optimal but also with the betweenness centrality metric. For each considered number of users, we remove a single node from the network according to the criticality metric under consideration, and we record the maximum aggregate utility of the resulting network. In order to appreciate the level of the cost function reduction achieved we also indicate the maximum aggregate utility value prior to node removal which we refer to as the original network. The incorrect selection of the critical node is reported as the maximum network utility, which in the considered scenario will be a node that creates a bottleneck for the network and thus bounds the maximum aggregate utility, such a node upon removal will render the network with a higher aggregate utility. The results are shown in Fig. 4. We observe that the proposed criticality metric yields smaller maximum aggregate utility values than the betweenness centrality metric which are close to the optimal values. This demonstrates the near optimality of the proposed solution.

\section{Computational Complexity}

It has been established in section III that the proposed change of sign approach is related to the sum of squared differences approach of equation (6) in the sense that nodes which lie in the sign cut-set report high sum of squared difference values. However, the main benefit of our approach is that the maximization algorithm does not have to be performed over the entire node set but only over the nodes which report the same sign of the Fiedler value element. In order to demonstrate, the significant reduction in computational effort achieved we compare the proposed algorithm in 1 against the maximization consensus algorithm proposed in [22] in terms of the computational time required for the algorithm to reach an equilibrium. In the simulation experiments that we conduct, we deploy nodes in an area of $1000 \times 1000 \mathrm{~m}^{2}$ with their $x$ and $y$ coordinates drawn from a uniform distribution. In order to evaluate the computational effort for different node densities and network sizes we consider number of node values in the range 100 to 1000 . Each node is assumed to have a fixed transmission radius of $250 \mathrm{~m}$. The computational time for the two approaches as a function of the number of nodes is shown schematically in Fig. 5. We observe that the proposed approach is able to achieve significant reductions in the computational time. These reductions become larger with increasing network size.

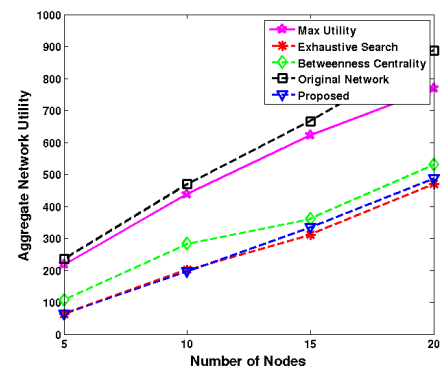

Figure 4. Aggregate network utility versus number of node in a network when critical nodes are selected using various approaches.

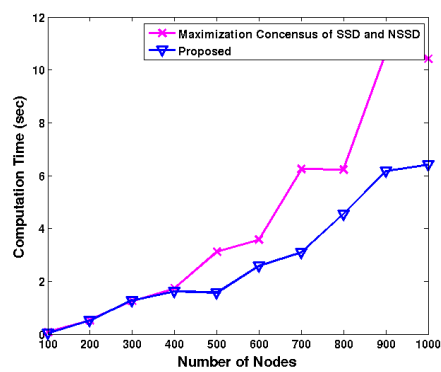

Figure 5. Computational time versus the number of nodes for the proposed approach and the maximization consensus algorithm of [22].

\section{Network Centric Evaluation}

Having established the suboptimality of the proposed solutions, and the significant reduction in implementation complexity achieved, in our final set of experiments we evaluate the performance of the proposed criticality metric in a more realistic network scenario. We conduct the simulation experiments on the Ns-3 Simulator [42] and evaluate the network performance using network centric performance criteria such as the total network throughput, the average per packet delay, the average per packet jitter and the total number of packets dropped. In all the simulations we compare the performance of the proposed metric against metrics such as, Betweenness Centrality, Closeness Centrality, Degree Centrality, Hybrid Interactive Linear Programming Rounding (HILPR), the Controllability of complex networks (Cont), the Sum Squared Difference (SSD) approach, the Normalized Sum Squared Difference (NSSD) approach and the previously proposed Spectral Partitioning for Node Criticality (SPNC) approach [27]. 


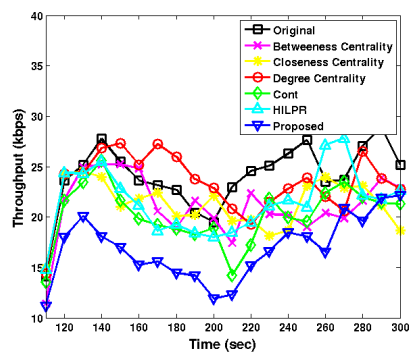

(a)

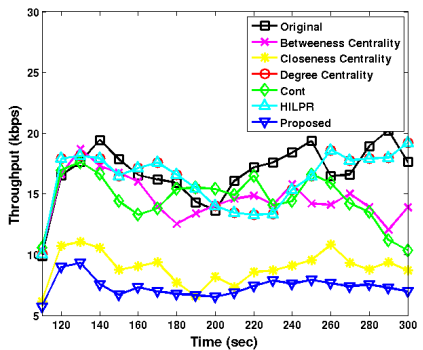

(c)

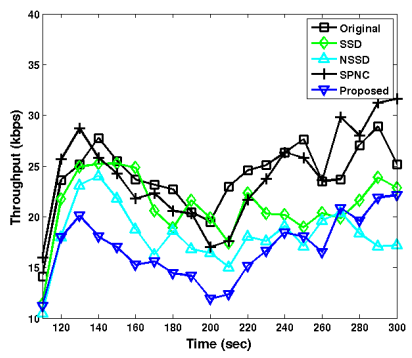

(b)

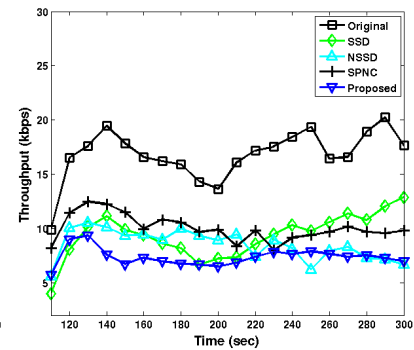

(d)
Figure 6. Time evolution of network throughput for the original network, and when a) \& b) A single node, c) \& d) $10 \%$ of the most critical nodes are removed according to betweeness centrality, closeness centrality, degree centrality, Hybrid Interactive Linear Programming Rounding (HILPR), the Controllability of complex networks (Cont),the Sum Squared Difference (SSD) approach, the Normalized Sum Squared Difference (NSSD) approach and the Spectral Partitioning for Node Criticality (SPNC) approach.

The evaluation was conducted on an area of $1500 \times 1500 \mathrm{~m}^{2}$, where 100 wireless adhoc network nodes were placed using a uniform random distribution. Each node was equipped with a $802.11 \mathrm{~b}$ transceiver with a transmit power of $7.5 \mathrm{dbm}$. $15 \%$ of them had an option of transmitting at a power $1.5 \times 7.5 \mathrm{dbm}$ [4] thus forming long range communication links. The degradation in signal strength as a function of the distance covered was represented by the Friss loss propagation model. A randomly selected set of 20 source/sink pairs initiate the communication in the network by transmitting packets at a rate of $2.048 \mathrm{~Kb} / \mathrm{s}$ each. Packet based transmission was assumed with the packet size set to 64byte packets. Routing paths within the network are formed using the OLSR (Optimized Link State Routing) protocol [43]. All measurements are obtained in the interval $100-300$ seconds after the start of the simulation. This provides sufficient time for the OLSR algorithm to converge to its equilibrium state. The degradation in network performance is evaluated after $10 \%$ of the most critical nodes are removed from the network. This process is repeated 10 times with the results averaged to decrease the stochastic uncertainty of the obtained results.

We first compare the performance of the proposed approach against the metrics under consideration in this paper for the network throughput that is achieved. The throughput of a network is defined as the total number of packets delivered to their destinations within the network per unit time. The main goal of any network configuration is to maximize the achieved throughput. In Fig 6a, 6b and Fig 6c, 6d we show the achieved

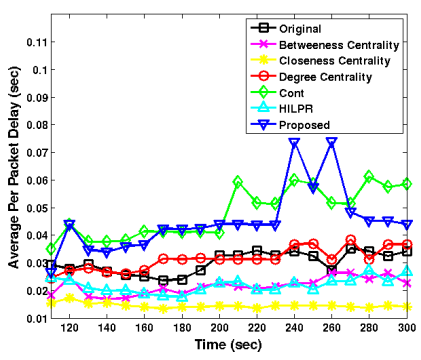

(a)

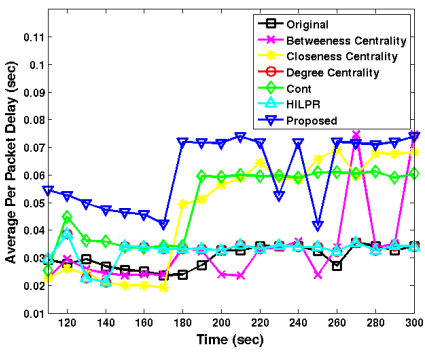

(c)

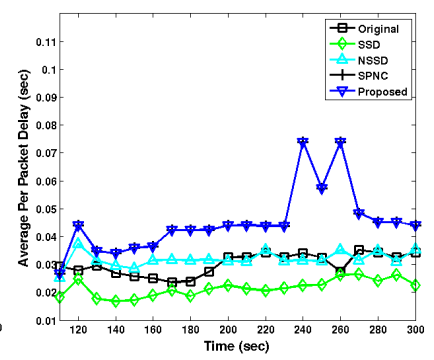

(b)

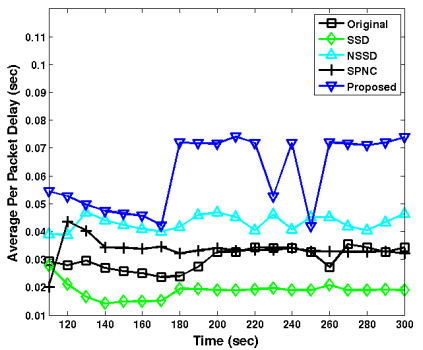

(d)
Figure 7. Time evolution of the per packet delay for the original network, and when a) \& b) A single node, c) \& d) $10 \%$ of the most critical nodes are removed according to betweeness centrality, closeness centrality, degree centrality, Hybrid Interactive Linear Programming Rounding (HILPR), the Controllability of complex networks (Cont), the Sum Squared Difference (SSD) approach, the Normalized Sum Squared Difference (NSSD) approach and the Spectral Partitioning for Node Criticality (SPNC) approach.

throughput after a single and $10 \%$ of the most critical nodes are removed from the network respectively. We observe that the proposed approach reports the highest decrease in the achieved throughput relative to the approaches that already exist in literature. This demonstrates that the proposed algorithm is successful in identifying the most critical nodes of a network. The decrease in average throughput observed at certain periods of time is due to the long range link which have a higher transmitter power compared to the rest of the nodes in the network. The increase in power enables them to cover a larger distance for relaying data and thus reserve a larger portion of the network, increasing the probability of collision in the network. This results in a similar trend observed by the original network and all the criticality metrics under consideration of a decrease in throughput at around $200 \mathrm{sec}$.

Next we conduct similar experiments, aiming at comparing the proposed criticality metric against other approaches using the average per packet delay of the network. The delay experienced by packets in transit is an important network attribute which describes its performance. Low delays are preferable. In wireless ad hoc networks, such as the one considered in this study, delays are due to a number of reasons: network congestion resulting in queuing delays, poor channel behaviour resulting in re-transmissions and contention resulting in large vacant medium delay times due to the CSMA/CA mechanism. In this work, we consider the average per packet delay as the performance metric. This is calculated by dividing the total number of delays observed with the number of packets 


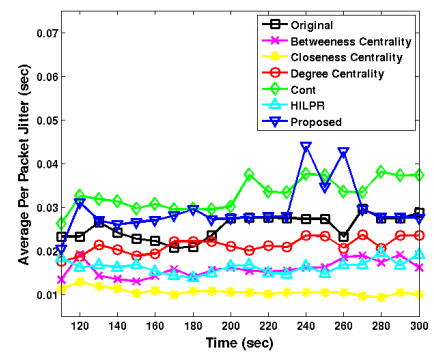

(a)

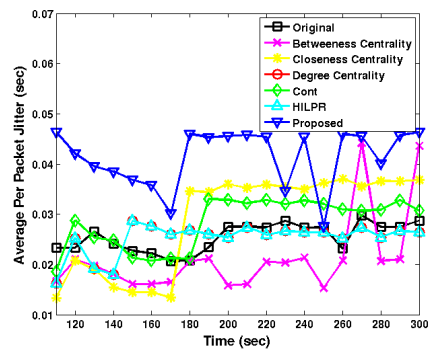

(c)

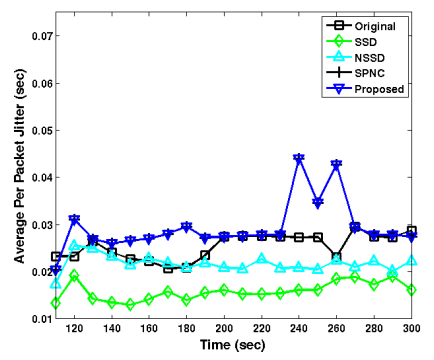

(b)

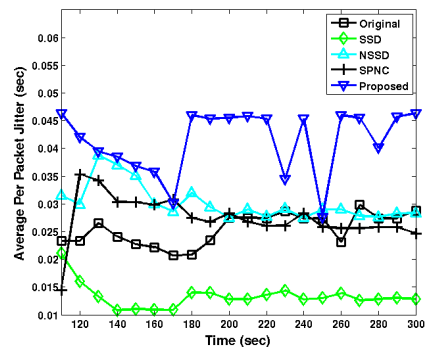

(d)
Figure 8. Time evolution of the per packet jitter for the original network, and when a) \& b) A single node, c) \& d) $10 \%$ of the most critical nodes are removed according to betweeness centrality, closeness centrality, degree centrality, Hybrid Interactive Linear Programming Rounding (HILPR), the Controllability of complex networks (Cont), the Sum Squared Difference (SSD) approach, the Normalized Sum Squared Difference (NSSD) approach and the Spectral Partitioning for Node Criticality (SPNC) approach.

transmitted throughout the simulation time. In Fig 7 we show the time evolution of the average per packet delay reported in the original network and when nodes are removed according to approaches that exist in literature and the proposed criticality metric. We observe that the proposed metric is able to bring a major degradation in performance as the average per packet delay increases significantly when nodes are removed. This is evidence of the fact that the proposed approach is more accurate in identifying the most critical nodes of a network.

We next consider the average per packet delay jitter as the performance metric. This is calculated by dividing the total delay jitter observed throughout the simulation experiment with the total number of transmitted packets. The delay jitter is calculated as the variation in packet reception times at the receiver. Increasing delay jitter values indicate increasing congestion within the network, so small delay jitter values are preferable. In Fig 8, we show the time evolution of the average per packet delay jitter observed in the original network and when nodes are removed according to various criticality metrics. We observe that the proposed metric outperforms the other proposals as it manages to significantly increase the delay jitter thus degrading network performance.

Finally, we consider the total number of dropped packets as the performance metric. High number of dropped packets in the network due to buffer overflow, is a strong indication of congestion. When nodes are removed from the network, the number of available paths decreases and the remaining paths are forced to accommodate all traffic. This makes them more

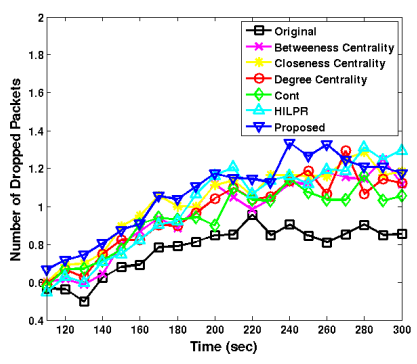

(a)

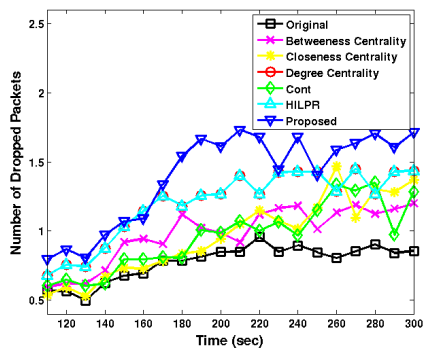

(c)

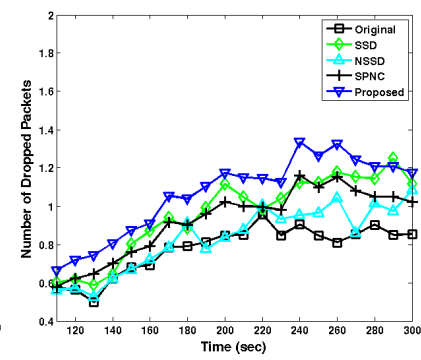

(b)

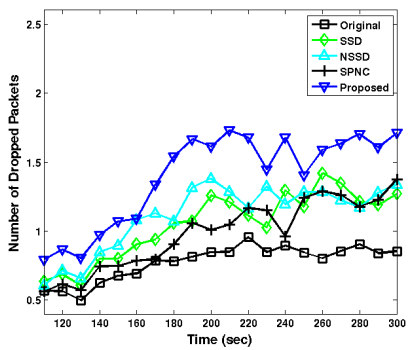

(d)
Figure 9. Time evolution of the number of dropped packets for the original network, and when a) \& b) A single node, c) \& d) $10 \%$ of the most critical nodes are removed according to betweeness centrality, closeness centrality, degree centrality, Hybrid Interactive Linear Programming Rounding (HILPR), the Controllability of complex networks (Cont), the Sum Squared Difference (SSD) approach, the Normalized Sum Squared Difference (NSSD) approach and the Spectral Partitioning for Node Criticality (SPNC) approach.

vulnerable to congestion. When critical nodes are removed, congestion is expected to be more severe and the number of dropped packets is thus higher. The results of the conducted simulation experiments are shown in Fig 9. We observe that during the whole simulation time the proposed scheme is able to bring a major increase in the number of dropped packets compared to other approaches thus making it a viable option for identifying critical nodes in a network.

\section{CONCLUSION}

In this work, we propose a new metric with which critical nodes can be identified in computer networks. We pose the problem in an optimization based framework and we develop the metric by combining suboptimal solutions of two optimization problems: the algebraic connectivity minimization problem which captures the topological aspects of node criticality and the min-max aggregate utility problem which captures its connection oriented nature. We show that the suboptimal solutions are not conservative and we demonstrate through extensive simulations the effectiveness of the proposed method and its superiority relative to other approaches. The method was evaluated on a wireless ad-hoc network. However, the problem formulation has been general and it thus opens the way for its application in other types of complex networks such as transportation networks, biological networks and water pipe networks. In the future, such extensions will be pursued in parallel with the development of a more efficient distributed 
algorithm that takes into account the change in the Fiedler vector elements across the network.

\section{REFERENCES}

[1] W. Asif, H. Qureshi, M. Rajarajan, and M. Lestas, "Cbdi: Combined banzhaf amp; diversity index for finding critical nodes," in IEEE Global Communications Conference (GLOBECOM) 2014, pp. 758-763, Dec 2014.

[2] M. Imran, M. A. Alnuem, M. S. Fayed, and A. Alamri, "Localized algorithm for segregation of critical/non-critical nodes in mobile ad hoc and sensor networks," Procedia Computer Science, vol. 19, pp. 11671172, 2013.

[3] M. Haenggi, "Energy-balancing strategies for wireless sensor networks," in Proceedings of the International Symposium on Circuits and Systems, 2003. ISCAS'03., vol. 4, pp. IV-828, IEEE, 2003.

[4] W. Asif, H. K. Qureshi, and M. Rajarajan, "Variable rate adaptive modulation (vram) for introducing small-world model into wsns," in Information Sciences and Systems (CISS), 2013 47th Annual Conference on, pp. 1-6, IEEE, 2013.

[5] A. Arulselvan, C. W. Commander, L. Elefteriadou, and P. M. Pardalos, "Detecting critical nodes in sparse graphs," Computers \& Operations Research, vol. 36, no. 7, pp. 2193-2200, 2009.

[6] S. Saroiu, P. K. Gummadi, and S. D. Gribble, "Measurement study of peer-to-peer file sharing systems," in Electronic Imaging 2002, pp. 156170, International Society for Optics and Photonics, 2001.

[7] Y. He, H. Ren, Y. Liu, and B. Yang, "On the reliability of large-scale distributed systems-a topological view," Computer Networks, vol. 53 no. 12, pp. 2140-2152, 2009.

[8] A. Arulselvan, C. W. Commander, P. M. Pardalos, and O. Shylo, "Managing network risk via critical node identification," Risk management in telecommunication networks, Springer, 2007.

[9] M. Kutz, Handbook of transportation engineering, vol. 768. McGrawHill New York, NY, USA:, 2004.

[10] Y. Shen, N. P. Nguyen, Y. Xuan, and M. T. Thai, "On the discovery of critical links and nodes for assessing network vulnerability," IEEE/ACM Transactions on Networking (TON), vol. 21, no. 3, pp. 963-973, 2013.

[11] R. Albert, I. Albert, and G. L. Nakarado, "Structural vulnerability of the north american power grid," Physical review E, vol. 69, no. 2, p. 025103 , 2004.

[12] P. Holme, B. J. Kim, C. N. Yoon, and S. K. Han, "Attack vulnerability of complex networks," Physical Review E, vol. 65, no. 5, p. 056109 . 2002.

[13] L. C. Freeman, "Centrality in social networks conceptual clarification," Social networks, vol. 1, no. 3, pp. 215-239, 1979.

[14] D. Bader, K. Madduri, et al., "Parallel algorithms for evaluating centrality indices in real-world networks," in International Conference on Parallel Processing ICPP., pp. 539-550, IEEE, 2006.

[15] Y.-Y. Liu, J.-J. Slotine, and A.-L. Barabási, "Controllability of complex networks," Nature, vol. 473, no. 7346, pp. 167-173, 2011.

[16] L. C. Freeman, "A set of measures of centrality based on betweenness," Sociometry, pp. 35-41, 1977.

[17] M. Everett and S. P. Borgatti, "Ego network betweenness," Social networks, vol. 27, no. 1, pp. 31-38, 2005.

[18] P. Bonacich, "Some unique properties of eigenvector centrality," Social Networks, vol. 29, no. 4, pp. 555-564, 2007.

[19] M. Fiedler, "Algebraic connectivity of graphs," Czechoslovak Mathematical Journal, vol. 23, no. 2, pp. 298-305, 1973

[20] P. Wei and D. Sun, "Weighted algebraic connectivity: An application to airport transportation network," in Proceedings of the 18th IFAC World Congress, Milan, Italy, 2011.

[21] P.-Y. Chen and A. O. Hero, "Local fiedler vector centrality for detection of deep and overlapping communities in networks," in International Conference on Acoustics, Speech and Signal Processing (ICASSP), pp. 1120-1124, IEEE, 2014
[22] H. Liu, X. Cao, J. He, P. Cheng, J. Chen, and Y. Sun, "Distributed identification of the most critical node for average consensus," in World Congress, vol. 19, pp. 1843-1848, 2014.

[23] T. Watanabe and N. Masuda, "Enhancing the spectral gap of networks by node removal," Physical Review E, vol. 82, no. 4, p. 046102, 2010.

[24] N. M. M. de Abreu, "Old and new results on algebraic connectivity of graphs," Linear algebra and its applications, vol. 423, no. 1, pp. 53-73, 2007.

[25] R. Merris, "Laplacian matrices of graphs: a survey," Linear algebra and its applications, vol. 197, pp. 143-176, 1994.

[26] A. Bertrand and M. Moonen, "Distributed computation of the fiedler vector with application to topology inference in ad hoc networks," Signal Processing, vol. 93, no. 5, pp. 1106-1117, 2013.

[27] W. Asif, M. Lestas, H. Qureshi, and M. Rajarajan, "Spectral partitioning for node criticality," in IEEE Symposium on Computers and Communications (accepted for publication), Jul 2015.

[28] F. Kelly, "Charging and rate control for elastic traffic," European transactions on Telecommunications, vol. 8, no. 1, pp. 33-37, 1997.

[29] M. Fiedler, "A property of eigenvectors of nonnegative symmetric matrices and its application to graph theory," Czechoslovak Mathematical Journal, vol. 25, no. 4, pp. 619-633, 1975.

[30] B. Mohar and Y. Alavi, "The laplacian spectrum of graphs," Graph theory, combinatorics, and applications, vol. 2, pp. 871-898, 1991.

[31] F. P. Kelly, A. K. Maulloo, and D. K. Tan, "Rate control for communication networks: shadow prices, proportional fairness and stability," Journal of the Operational Research society, pp. 237-252, 1998.

[32] D. P. Palomar and M. Chiang, "Alternative distributed algorithms for network utility maximization: Framework and applications," IEEE Transactions on Automatic Control, vol. 52, no. 12, pp. 2254-2269, 2007.

[33] X. Xiaochun and W. Xiaoyan, "A simple rate control algorithm for maximizing total user utility," in Proceedings of the International Conference on Communication Technology, pp. 135-138, 2003.

[34] P. Christiano, J. A. Kelner, A. Madry, D. A. Spielman, and S.-H. Teng, "Electrical flows, laplacian systems, and faster approximation of maximum flow in undirected graphs," in Proceedings of the forty-third annual ACM symposium on Theory of computing, pp. 273-282, ACM, 2011.

[35] M. Lestas, A. Pitsillides, P. Ioannou, and G. Hadjipollas, "A new estimation scheme for the effective number of users in internet congestion control," IEEE/ACM Transactions on Networking (TON), vol. 19, no. 5, pp. 1499-1512, 2011.

[36] D. A. Spielman and N. Srivastava, "Graph sparsification by effective resistances," SIAM Journal on Computing, vol. 40, no. 6, pp. 19131926, 2011.

[37] A. Ghosh and S. Boyd, "Growing well-connected graphs," in 45th IEEE Conference on Decision and Control, pp. 6605-6611, IEEE, 2006.

[38] G. H. Golub, "Some modified matrix eigenvalue problems," Siam Review, vol. 15, no. 2, pp. 318-334, 1973.

[39] W. H. Haemers, "Interlacing eigenvalues and graphs," Linear Algebra and its applications, vol. 226, pp. 593-616, 1995.

[40] O. Rojo, R. Soto, and H. Rojo, "Bounds for sums of eigenvalues and applications," Computers \& Mathematics with Applications, vol. 39, no. 7 , pp. $1-15,2000$

[41] MathWorks, "Matlab 2013," http://www.mathworks.com /support /sysreq /sv-r2013a/.

[42] NSNAM, "Network simulator 3," https://www.nsnam.org/.

[43] C. Mbarushimana and A. Shahrabi, "Comparative study of reactive and proactive routing protocols performance in mobile ad hoc networks," in 21st International Conference on Advanced Information Networking and Applications Workshops, vol. 2, pp. 679-684, IEEE, 2007. 